Revista de la Escuela de Ciencias de la Educación. 2022, Año 18 1(17) 18-31. Enero a junio. Anderete Schwal, M., La pandemia y el año que enseñamos por WhatsApp: el recurso tecnológico más utilizado en las secundarias pobres de Bahía Blanca durante el 2020.

\title{
LA PANDEMIA Y EL AÑO QUE ENSEÑAMOS POR WHATSAPP: EL RECURSO TECNOLÓGICO MÁS UTILIZADO EN LAS SECUNDARIAS POBRES DE BAHİA BLANCA DURANTE EL 2020
}

\author{
THE PANDEMIC AND THE YEAR WE TEACH ON WHATSAPP: THE MOST USED \\ TECHNOLOGICAL RESOURCE IN POOR HIGH SCHOOLS IN BAHIA BLANCA \\ DURING 2020
}

\begin{abstract}
Mariano Anderete Schwal
Universidad Nacional del Sur - CONICET, Argentina

marianoand3@hotmail.com
\end{abstract}

Recibido: 30 de julio de 2021

Aprobado: 24 de octubre de 2021

Publicado: 31 de diciembre de 2021

Cita sugerida: Anderete Schwal, M. (2022). La pandemia y el año que enseñamos por WhatsApp: el recurso tecnológico más utilizado en las secundarias pobres de Bahía Blanca durante el 2020. Revista de la Escuela de Ciencias de la Educación. 1(17), 18-30.

\section{RESUMEN}

La aparición de la Covid-19 en todo el mundo generó un cierre masivo de escuelas durante el año 2020, no obstante, el personal docente continuó enseñando mediante la educación a distancia, adaptando diversas estrategias según sus posibilidades. El presente trabajo analiza las consecuencias educativas de la pandemia en la educación secundaria argentina durante su primer año y cuáles fueron las Tecnologías de la Información y la Comunicación (TIC) utilizadas por los docentes y el alumnado, en un contexto de segregación educativa. La investigación consiste en un estudio descriptivo utilizando un método cualitativo a partir de entrevistas a docentes y directivos de escuelas pertenecientes al nivel secundario de la ciudad de Bahía Blanca, a quienes se les indagó sobre percepciones y experiencias en torno a los distintos métodos tecnológicos de educación utilizados durante el año escolar 2020. Los resultados de la investigación dan cuenta de una relación entre las condiciones socioeconómicas del alumnado y las herramientas utilizadas por sus profesores. En especial se destaca el uso de la aplicación WhatsApp, la cual se reduce a la única tecnología disponible para la mayoría de los estudiantes que concurren a los colegios secundarios más pobres, ya que para ellos el celular fue su único medio de conexión educativa. 
Revista de la Escuela de Ciencias de la Educación. 2022, Año 18 1(17) 18-31. Enero a junio. Anderete Schwal, M., La pandemia y el año que enseñamos por WhatsApp: el recurso tecnológico más utilizado en las secundarias pobres de Bahía Blanca durante el 2020.

Palabras clave: Segregación educativa - WhatsApp - Brecha digital - TIC COVID-19.

\section{ABSTRACT}

The appearance of Covid-19 around the world generated a massive closure of schools during 2020, however the teaching staff continued teaching through distance education, adapting various strategies according to their possibilities. This paper analyzes the educational consequences of the pandemic in Argentine secondary education during its first year and what Information and Communication Technologies (ICT) were used by teachers and students, in a context of educational segregation. The research consists of a descriptive study using a qualitative method based on interviews with teachers and directors of the secondary level of the city of Bahía Blanca, who were asked about perceptions and experiences around the different technological methods of education used during the study. school year 2020. The results of the research reveal a relationship between the socioeconomic conditions of the students and the tools used by their teachers. In particular, the use of the WhatsApp application stands out, which is reduced to the only technology available to the majority of students who attend the poorest secondary schools, since for them the cell phone was their only means of educational connection.

Keywords: Educational segregation - WhatsApp - Digital divide - ICT - COVID19.

\section{INTRODUCCIÓN}

La aparición de la pandemia por COVID-19 en el año 2020 generó que la mayoría de los países del mundo cerraran las escuelas por ser posibles focos de contagio y tuvieran que desarrollar estrategias alternativas para asegurar la continuidad educativa. Ante la llegada del coronavirus al país, el presidente de Argentina determinó mediante Decreto 297/2020 el ASPO (Aislamiento Social Preventivo y Obligatorio) de todos los ciudadanos, a los efectos de disminuir la curva de contagios del nuevo virus. En el ámbito educativo, se suspendieron las clases presenciales en todas las escuelas del país y tal situación presentó un desafío para las autoridades nacionales respecto de poder continuar con la educación de millones de niños, niñas y adolescentes. Entonces oficialmente se desarrolló un proyecto llamado "Seguimos Educando", por el cual se utilizaron principalmente recursos virtuales a cargo del Ministerio de Educación de la Nación destinados a asegurar la continuidad educativa a distancia. Pero dicho recurso era de aplicación voluntaria para los educadores y en la práctica no tuvo éxito (Anderete Schwal, 2020; Romero, Krichesky y Zacarías, 2021). Entonces, escuelas y docentes utilizaron sus propias estrategias educativas de acuerdo a los recursos disponibles de sus estudiantes y sus familias, que variaron significativamente según el tipo de escuela a la que asisten. Esto sucedió por la marcada desigualdad socio económica entre los estudiantes que concurren a los distintos tipos de 
Revista de la Escuela de Ciencias de la Educación. 2022, Año 18 1(17) 18-31. Enero a junio. Anderete Schwal, M., La pandemia y el año que enseñamos por WhatsApp: el recurso tecnológico más utilizado en las secundarias pobres de Bahía Blanca durante el 2020.

escuela, lo cual configura una importante segregación educativa en todo el país (Veleda, 2014).

En la Argentina contemporánea existe una sociedad fragmentada y con mucha desigualdad socio económica (Kessler, 2011). En el plano educativo se observa una reproducción de dicha desigualdad, por cuanto reciben más los que más tienen (Llach, 2006), relegando a los que menos tienen a estudiar en escuelas pobres para pobres (Krüger, 2012). Estos últimos no cuentan con recursos digitales suficientes para desarrollar la educación a distancia pretendida por las políticas educativas públicas, reproduciéndose la desigualdad educativa mediante una brecha digital según los recursos tecnológicos de los distintos sectores socio económicos (Vivanco, 2020).

Las Tecnologías de la Información y las Comunicaciones (TIC) se consideran un complemento de la educación presencial, pero a partir de la cuarentena por COVID-19 se convirtieron en esenciales para las clases a distancia. Los docentes pueden utilizar diferentes plataformas y herramientas tecnológicas para procesar toda la información que se requiera para realizar las actividades y comunicarse con sus alumnos. Los recursos se ajustan a los recursos tecnológicos disponibles, pudiendo utilizarse computadoras, tablets, televisores, teléfonos celulares, entre otros dispositivos electrónicos.

WhatsApp es una aplicación de mensajería gratuita que usa la conexión a Internet del teléfono móvil para enviar mensajes (y así evitar cargos de SMS), teniendo funciones muy útiles para ser utilizadas en el ámbito educativo como son: realizar video llamadas, crear grupos y compartir entre sus integrantes un número ilimitado de archivos (textos, imágenes, videos y mensajes de audio, entre otros). ${ }^{1}$ Por tales ventajas y al requerir mínimamente un teléfono celular, se convirtió en el medio más utilizado para que los docentes se comuniquen con sus alumnos y puedan compartir sus clases, al menos entre aquellos que no tenían acceso a una computadora. Una investigación sobre la educación argentina en tiempos de pandemia (Nadorowski, Volman y Braga, 2020) da cuenta de que el WhatsApp fue el medio más utilizado para la propuesta de tareas, el $80 \%$ de los docentes respondió que las escuelas siempre lo utilizan, asimismo sostienen que el $57,7 \%$ utilizó el envío de archivos PDF o Word para tales efectos. Los autores sostienen que "si pudiésemos definir cómo se construye la escena educativa en la Argentina de la pandemia la respuesta, claramente, es el WhatsApp" (2020, p. 8). A su vez, el personal docente y directivo de las escuelas empleó los grupos de dicha aplicación para organizar su labor durante la pandemia, ya que no podían concurrir presencialmente a la escuela. Dussel y Fuentes Cardona (2021) mencionan que los chats de estos grupos se presentaron como círculos motivaciones y carteleras de mensaje para los docentes, a modo de coordinar el trabajo durante la no presencialidad por pandemia.

En la presente investigación cualitativa se analiza la utilización de recursos tecnológicos durante el primer año de la pandemia por parte de docentes de diversas escuelas del nivel secundario en la ciudad de Bahía Blanca, Argentina. Se destaca aquel recurso más utilizado en los sectores más pobres fue la

${ }^{1}$ https://www.whatsapp.com/features/ 
Revista de la Escuela de Ciencias de la Educación. 2022, Año 18 1(17) 18-31. Enero a junio. Anderete Schwal, M., La pandemia y el año que enseñamos por WhatsApp: el recurso tecnológico más utilizado en las secundarias pobres de Bahía Blanca durante el 2020.

aplicación WhatsApp para teléfonos celulares, seguido por el Google Classroom y los correos electrónicos. Se estudia su puesta en práctica por parte de los docentes y los motivos por los cuales fue ampliamente usado en las escuelas ubicadas en los sectores más pobres de la ciudad.

\section{DESARROLLO}

\section{Antecedentes teóricos y contexto educativo}

Los primeros estudios sobre segmentación educativa en Argentina se remontan a Braslavsky (1985) y posteriormente a los trabajos de Kessler (2002), Tiramonti (2004), Veleda (2014), y recientemente Krüger (2019). Las investigaciones mencionadas demuestran una significativa distancia de acceso a la educación de calidad según sector social de pertenencia. Además de este acceso diferencial, se evidencian los circuitos y recorridos diversos que realizan los estudiantes en sus trayectorias educativas según su pertenencia social y económica. Asimismo, se replican las diferencias entre sectores en el acceso a los recursos digitales de información y comunicación necesarios para la educación a distancia durante la pandemia por COVID-19. Dussel (2020) indica que esta situación ha generado la "domiciliación" de lo escolar, trasladándola hacia el espacio doméstico. En dicho ámbito se deben contar con las Tecnologías de la Información y Comunicación (TIC) suficientes para poder desarrollar las clases a distancia propuestas por el gobierno nacional, de lo contrario la equidad educativa se verá afectada. Para estudiar en casa se requiere contar con una computadora, una tablet $o$ un teléfono celular, acceso a internet, software determinado, conocimientos previos para utilizar dicha tecnología y tiempo disponible. A estos recursos digitales se le debe sumar la presencia de un adulto que oriente a los adolescentes que intenten utilizarlos en caso de no saber cómo hacerlo. Pero no todos los estudiantes en Argentina disponen de los mismos recursos digitales, existiendo una marcada desigualdad de acuerdo a la situación socioeconómica de cada familia (Formichella y Krüger, 2020). La educación a distancia desarrollada durante de la cuarentena refleja una escolarización diferencial del alumnado en función de la clase social de pertenencia, tipo de escuela, zona de residencia, región y territorio (Cabrera, Pérez y Santana, 2020).

La Brecha digital es un concepto creado para definir las desigualdades en el acceso y uso de las TIC. Pimienta (2007) afirma que es el reflejo de la brecha social en el mundo digital y puede definirse en términos de la desigualdad de posibilidades que existen para acceder a la información, al conocimiento y la educación mediante las nuevas tecnologías. Por su parte Martínez López (2020) sostiene que la brecha digital "es la expresión de la desigualdad en el uso y acceso de las nuevas Tecnologías de la Información y la Comunicación (TIC), y como desigualdad es generadora de procesos de exclusión social" (p. 1). Esta situación se evidencia en las diferencias en el acceso a recursos tecnológicos y a 
Revista de la Escuela de Ciencias de la Educación. 2022, Año 18 1(17) 18-31. Enero a junio. Anderete Schwal, M., La pandemia y el año que enseñamos por WhatsApp: el recurso tecnológico más utilizado en las secundarias pobres de Bahía Blanca durante el 2020.

la conexión a internet, especialmente en los países latinoamericanos (CEPAL, 2020):

Esta brecha digital puede reproducirse a través del sistema educativo de diversas maneras, ya que tanto los estudiantes, como los profesores y las familias, cuentan con dificultades y limitaciones que impiden que las competencias tecnológicas y el acceso a recursos educativos se dé en condiciones igualitarias que permitan disminuir la brecha no sólo digital, sino también educativa (Anderete Schwal, 2021, p.46).

Los organismos internacionales como UNESCO (2020) y UNICEF (2020) indican que además de la brecha digital entre clases sociales, existe una brecha familiar. Por tal motivo los obstáculos no son sólo digitales por la carencia de dispositivos electrónicos, sino que las familias con menor educación tienen más dificultades para ayudar a sus estudiantes en las tareas escolares en casa, produciéndose así un aumento de la segregación educativa en este contexto de cuarentena. Esta brecha digital puede reproducirse a través del sistema educativo de diversas maneras, ya que tanto los estudiantes, como los profesores y las familias, cuentan con dificultades y limitaciones que impiden que las competencias tecnológicas y el acceso a recursos educativos se dé en condiciones igualitarias que permitan disminuir la brecha no sólo digital, sino también educativa.

En el caso de Argentina existe un desigual acceso a recursos tecnológicos con conexión a internet según el nivel socio económico (NSE) de las familias, en tanto que los estudiantes de secundaria de bajo NSE con computadora en sus viviendas son del $54,85 \%$, mientras que los de alto NSE alcanzan un $99,03 \%$. No obstante, respecto de la tenencia de teléfonos celulares la brecha no es tan marcada, ya que el $91,80 \%$ de los estudiantes con bajo nivel cuentan con uno, así como el $98,93 \%$ de nivel alto (Cardini, D'Alessandre y Torre, 2020). Los teléfonos celulares se convirtieron en el medio de comunicación más extendido en el mundo, superando a la prensa escrita, la televisión y a las computadoras (Díaz Jatruf, 2014). En tal sentido, los celulares son el recurso tecnológico más popular en Argentina, al cual la mayoría de los estudiantes tiene acceso y para gran parte de ellos es su única conexión educativa. Al utilizar una conexión a internet su funcionalidad se multiplica, ya que se puede navegar por la web y descargar distintos tipos de aplicaciones que pueden utilizarse con fines educativos, siendo las más utilizadas por los docentes el WhatsApp y la plataforma de Google Classroom.

Aquellas escuelas con mayores recursos económicos cuentan con plataformas educativas propias online y sus estudiantes con conexión a internet. En cuanto a los medios para proponer tareas, el $80,5 \%$ de las familias de escuelas privadas utilizan plataformas de videoconferencia o de streaming (como Zoom, Meet o Skype) para dar clases sincrónicas, mientras que la proporción desciende 
Revista de la Escuela de Ciencias de la Educación. 2022, Año 18 1(17) 18-31. Enero a junio. Anderete Schwal, M., La pandemia y el año que enseñamos por WhatsApp: el recurso tecnológico más utilizado en las secundarias pobres de Bahía Blanca durante el 2020.

al $29,4 \%$ en las instituciones estatales, que se inclinan hacia la educación asincrónica. Asimismo, la herramienta más utilizada en las escuelas estatales es el WhatsApp (92,2\% lo utilizan siempre o a veces), que se ubica recién en el $6^{\circ}$ puesto entre las más usadas en las escuelas privadas $(68,0 \%)$, por detrás de las aplicaciones para clases sincrónicas, los archivos PDF o Word y los libros de texto (Narodowski, Volman y Braga, 2020).

Las investigaciones previas sobre la brecha digital en pandemia destacan que las escuelas privadas, las cuales responden a un estudiantado con un mayor nivel socioeconómico, cuentan con mayores recursos tecnológicos para desarrollar las clases a distancia en forma sincrónica y con la utilización plena de internet mediante el uso de computadoras. Mientras que, en las escuelas públicas de la periferia, que apuntan a un sector más humilde de la población, los docentes dictan clases asincrónicas y les dejan el material a sus estudiantes en grupos de WhatsApp o en formato papel.

\section{Metodología}

El estudio que se presenta es de carácter descriptivo y cualitativo, el cual pretende investigar los recursos tecnológicos utilizados por los docentes del nivel secundario, en un contexto de segregación educativa y su correlativa brecha digital. A tales efectos se entrevistaron a docentes y directivos del nivel secundario de la ciudad Bahía Blanca, provincia de Buenos Aires. Se utiliza un muestreo no probabilístico y por cuotas (Hernández Sampieri; Fernández Collado y Baptista Lucio, 2014), a los efectos de tomar a las escuelas más significativas de cada sector socio educativo de la ciudad. En tal sentido, la muestra se estructura a partir de una heterogeneidad socio educativa que también caracteriza al resto de las ciudades medianas y grandes del país. La selección de establecimientos educativos se realiza a partir de los criterios clásicos para el análisis de la segmentación educativa (Braslavsky, 1985). Siguiendo la tradición de los estudios del campo de la sociología de la educación, se distinguen tres segmentos (bajo, medio, alto) de acuerdo a las características socioeconómicas de la población que asiste (Kessler, 2002), de tal forma se entrevistó a directivos y personal docente que trabajan en distintos contextos educativos (Pintrich y Schunck, 2006). Se entrevistó en total a 16 directivos y 20 docentes del nivel secundario de Bahía Blanca, a modo de abarcar las distintas realidades socio económicas comprendidas por las variables mencionadas. En función de los antecedentes disponibles, se agrupa a las instituciones en dos segmentos: de gestión estatal y privada. Clasificando a las primeras según su ubicación geográfica, de acuerdo a los estudios que vinculan la desigualdad socioeducativa con la segregación residencial (Formichella y Krüger, 2019), de tal manera se clasifican en escuelas públicas céntricas y periféricas. En cuanto a las escuelas de gestión privada, se las clasifican según su ubicación, coincidiendo las céntricas como aquellas con menor subvención estatal y con mayor nivel socioeconómico de sus estudiantes. A su vez, las escuelas preuniversitarias locales son públicas, pero se consideran parte del grupo socio económico medio y alto, conforme las características de su alumnado (Anderete Schwal, 2019). 
Las entrevistas se realizaron mediante video llamadas a mediados del año 2020 y comienzos del 2021. A tales efectos, se elaboró un cuestionario semi estructurado que comprende aspectos relevantes para el presente estudio. La entrevista semiestructurada es una de las técnicas más utilizadas en la metodología cualitativa (Flick, 2012), en ellas se desarrolla una conversación entre dos o más personas, partiendo de unas preguntas previamente seleccionadas. El guion utilizado se encontraba conformado por preguntas abiertas y con respuestas a desarrollar vinculadas con la educación en el nivel secundario durante el primer año de la pandemia. Una vez transcriptas la totalidad de las entrevistas, se procedió a trabajar con las respuestas conforme las categorías de análisis adecuadas a la teoría utilizada. De tal manera, se tomaron las siguientes categorías: ubicación residencial de la escuela; nivel socio económico de los estudiantes; recursos tecnológicos disponibles por parte de los docentes y sus estudiantes; dictado de las clases no presenciales utilizando dichos recursos tecnológicos; y el uso en particular del WhatsApp como herramienta educativa. A partir de allí, el método comparativo fue la base del análisis y pretendió develar las similitudes y los contrastes entre las escuelas públicas y privadas, en el objetivo de identificar la forma en la cual dictaron las clases no presenciales según sus condiciones socioeconómicas y de qué forma se utilizó la aplicación WhatsApp.

A continuación, se presentan los resultados obtenidos a partir de las declaraciones recogidas en las entrevistas, las cuales dan cuenta de la diversidad de situaciones presentadas en las distintas escuelas secundarias de Bahía Blanca durante la pandemia, en un contexto de segregación educativa vigente durante el año 2020.

\section{Resultados}

A partir de las entrevistas realizadas, se organizan las respuestas en torno a distintos ejes que representan las problemáticas recurrentes mencionadas en torno a la utilización de los recursos tecnológicos desarrollados durante la pandemia. De tal manera se reflejan diversas situaciones descriptas por el personal directivo y docente entrevistado, dependiendo del tipo de escuela donde trabajan.

\section{Diferencias de recursos tecnológicos entre escuelas según su gestión a) Escuelas de alto nivel socio económico:}

Las escuelas de gestión privada son las que cuentan con un mayor nivel socioeconómico entre su alumnado, en especial aquellas sin subvención estatal. Estas instituciones tienen plataformas digitales propias anteriores a la pandemia y sus estudiantes cuentan -en su totalidad- con computadoras y acceso a internet. En la misma situación se encuentran las escuelas preuniversitarias de la ciudad de Bahía Blanca, las cuales atienden a sectores económicos similares. Estos colegios contaron con los recursos tecnológicos suficientes para poder continuar con la enseñanza a distancia. 
Revista de la Escuela de Ciencias de la Educación. 2022, Año 18 1(17) 18-31. Enero a junio. Anderete Schwal, M., La pandemia y el año que enseñamos por WhatsApp: el recurso tecnológico más utilizado en las secundarias pobres de Bahía Blanca durante el 2020.

Nosotros tenemos lo siguiente: Una plataforma de la editorial Santillana, que lo usa todo primaria, primer año, segundo y tercero del secundario. Cuarto, quinto y sexto tiene otra plataforma que es "colegium", pero después usan el Zoom complementariamente para comunicarse con sus alumnos (Directivo 1, Escuelas privada céntrica, sin subvención estatal, atiende a clases altas).

Usamos la plataforma propia de la escuela y las complementamos con clases sincrónicas por zoom. Le pedimos a los alumnos que tengan las cámaras prendidas, así podemos controlar que estén prestando atención a la clase. La realidad es que todos tienen computadoras y acceso a internet, eso permite enfocarte en organizar tu clase (Docente 3, escuela privada céntrica, 60\% subvención estatal, atiende a clases altas).

Usamos la plataforma que usa la universidad, que es el moodle. Este año algunos docentes tuvieron que capacitarse para poder usarlo. De parte de los alumnos no tuvimos problemas porque todos tienen conectividad desde sus computadoras o sus celulares (Directivo 3, escuela preuniversitaria, atiende a sectores medios altos).

En estas escuelas se destaca la utilización de clases a distancia sincrónicas, es decir los profesores se comunican en tiempo real con sus alumnos a través de aplicaciones como Zoom o Google Meet, las cuales son complementadas por plataformas digitales propias de cada institución educativa, donde los docentes cargan el contenido teórico y las actividades a desarrollar.

\section{b) Escuelas de nivel socioeconómico medio y bajo:}

La brecha digital se evidencia en las escuelas secundarias públicas, donde las familias de los estudiantes que provienen de la periferia pobre de la ciudad (Chavez, 2017) cuentan con menores recursos económicos que el resto y ello se refleja en un peor acceso a los recursos tecnológicos necesarios para la educación a distancia, lo cual repercute en las estrategias educativas de sus docentes.

Hay profes que trabajan con Classroom, pero bueno la realidad es que no todos los chicos tienen acceso al Classroom. Entonces se implementó el mail, después los grupos de WhatsApp, que es lo que más funciona. Lo que más manejan los chicos y los profes son los grupos de WhatsApp. Después vinieron los cuadernillos para aquellos que no tenían conectividad, pero los cuadernillos proponen actividades que no son las mismas que están desarrollando los profesores. Entonces descartamos los cuadernillos y estamos trabajando con una fotocopiadora que está a la vuelta de la escuela, entonces los chicos que no tienen conectividad van y buscan las tareas en la fotocopiadora (Directivo 5, escuela pública periferia, atiende a clases bajas).

No todos los estudiantes provenientes de los barrios más pobres cuentan con una computadora en sus hogares, en cambio sí cuentan con al menos un teléfono celular en sus familias. Por ello los profesores se comunicaron mediante la aplicación WhatsApp y desarrollaron su tarea docente a través de dicha tecnología. Supletoriamente se utilizó el recurso Classroom de Google, el cual fue 
Revista de la Escuela de Ciencias de la Educación. 2022, Año 18 1(17) 18-31. Enero a junio. Anderete Schwal, M., La pandemia y el año que enseñamos por WhatsApp: el recurso tecnológico más utilizado en las secundarias pobres de Bahía Blanca durante el 2020.

organizado por directivos y docentes de numerosas escuelas públicas secundarias. En dicha plataforma cada curso tiene un "aula virtual", donde los docentes de cada materia se comunican con sus alumnos, les dejan el material y suben las tareas a realizar. Pero el éxito de esta alternativa depende de la conectividad de los estudiantes, la cual no siempre está presente. Por otra parte, se presentan casos donde hay alumnos que no tienen ningún tipo de conectividad, ya que ni siquiera tienen un teléfono celular en sus casas. Ante esa situación los docentes debieron utilizar el clásico recurso del papel, por lo que dichos alumnos debían acercarse a las escuelas para retirar las clases impresas, coincidiendo en la mayor parte de las veces con la entrega de bolsones de comida.

La entrega de los bolsones de comida las hacíamos los docentes que nos acercábamos a la escuela en forma voluntaria, eran alumnos y alumnas que no los conocíamos porque estuvieron ausentes durante todo el año, pero se notaba que sus familias necesitaban esa comida. Entonces aprovechamos para entregarles el cuadernillo del gobierno para que se llevaran algo para estudiar. Aunque en nuestras clases no los usamos porque no se ajusta a los contenidos que desarrollamos, pero lo importante era alentarlos para que sigan estudiando (Docente 2, escuela pública de la periferia, atiende a clases bajas).

En las escuelas públicas de los barrios más pobres, la entrega de bolsones de comida cada quince días para los estudiantes que solían utilizar el comedor escolar se convirtió en una fuente de alimento para toda su familia. A medida que se prorrogaba el ASPO, se fueron sumando más pedidos de bolsones. Este momento se aprovechó para entregarles los cuadernillos impresos del programa "seguimos educando" a quienes no contaban con la conectividad necesaria para realizar las clases virtuales a distancia. Aunque dicho recurso no fue aplicado por los docentes entrevistados, sino que para dejarles las clases a sus alumnos recurrieron a las fotocopias y entrega de las tareas en formato de papel.

\section{Detalles de la utilización del WhatsApp en las escuelas}

Durante el año 2020, las clases no presenciales de las escuelas públicas se dictaron en forma asincrónica, el recurso más utilizado fue la aplicación WhatsApp para celulares y se utilizaron archivos con extensión PDF o Word (Narodowski, Volman y Braga, 2020). La aplicación fue ampliamente utilizada por los docentes de Argentina, tanto para el personal de las escuelas con fines organizativos, como para comunicarse con los estudiantes y garantizar dictado de las clases virtuales.

La primera forma de utilizarlo fue universal para los docentes de todas las escuelas, ya que la mayoría de ellos pertenece a clases medias (Kessler, 2002) y cuentan con dicha aplicación como herramienta diaria. La segunda forma fue más utilizada en las escuelas de clase media y baja, ya que al preparar los métodos de enseñanza y comunicación debieron adaptarse a las diversas características de cada escuela y su alumnado. Ante la falta de computadoras y medios sincrónicos de comunicación, debieron optar por el WhatsApp como primer recurso comunicativo. 
Revista de la Escuela de Ciencias de la Educación. 2022, Año 18 1(17) 18-31. Enero a junio. Anderete Schwal, M., La pandemia y el año que enseñamos por WhatsApp: el recurso tecnológico más utilizado en las secundarias pobres de Bahía Blanca durante el 2020.

En mis clases usamos WhatsApp. Primero yo mandaba un trabajo por escrito, en PDF porque ellos siempre pedían PDF, porque otros archivos no podían abrir. Ellos lo leían y hacían las consultas en la clase siguiente. En el horario de clase, pero no se respetaban los horarios, porque en realidad trabajaba todos los días, aunque solo me correspondía dos veces por semana. Si no entendían algo, que me pasó mucho, hacíamos videollamada particular con ese alumno. Con fotos me mandaban las tareas, por correo nada, era o por foto o por un documento con extensión rarísima. Se ve que lo hacían en el celular. Con la foto la abría en la compu con WhatsApp web, lo copiaba y lo abría con Paint para poder ampliarla y vela mejor (Docente 2, escuela pública de la periferia, atiende a clases bajas).

La mayoría usa WhatsApp porque es más fácil de usar y además permite llegar a todos los alumnos. Las escuelas más preparadas usan otro sistema como Google Classroom o los mails. Pero el problema es que no todos tienen una computadora en sus casas, en cambio sí tienen celular y por eso prefieren el WhatsApp. Pero también tiene sus desventajas, te escriben a cualquier hora y tienen tu número personal, los profesores no podemos tener un celular para la escuela y otro para la familia, todos tenemos el mismo y te cuesta despegarte de la escuela, no podés mirar para otro lado. Tenés que estar disponible todo el día (Docente 5, escuela pública de la periferia, atiende a clases bajas).

Los docentes de escuelas periféricas consultados dan cuenta de la utilización del WhatsApp como el recurso tecnológico más popular y que alcanza a la mayoría de sus estudiantes. No obstante, mencionan dificultades tecnológicas que debieron superar para que dicho recurso funcione en todos los casos: a) Debieron utilizar un formato de archivo que sea de fácil acceso y compatible con todos los teléfonos celulares, utilizando archivos con extensión PDF; y b) Recibían sus tareas a través de fotografías que no siempre tenían buena definición. Esto se debe a que, si bien casi la totalidad de los estudiantes utiliza teléfonos celulares, no significa que sepan cómo trabajar con todas sus funciones. Aunque la mayoría de los adolescentes se encuentran familiarizados con esta tecnología, la destinan principalmente para redes sociales y videojuegos, y no para las actividades escolares (Gewerc, Fraga y Rodés, 2017).

Las preceptoras tenían los teléfonos de WhatsApp de los profesores y de los chicos. Entonces se habló con los profesores, también hay que entender que el teléfono es privado, bueno con los profesores que no se animaban a dar su teléfono comprando un chip de 50 pesos podían tener un número de WhatsApp para los alumnos, lo que se ahorraban en el colectivo, en combustible. La mayoría dio su teléfono, no tuvo drama, algunos tienen el mail. Se organizó bastante rápido. Sí costó, costó. Porque había que tener a todos enganchados, nosotros necesitábamos tener a todos conectados, vinculados (Directivo 7, escuela pública de la periferia, atiende a clases bajas).

El personal docente de las escuelas públicas menciona que su tiempo de trabajo se extendió durante la educación no presencial, puesto que debían estar todo el día disponible para comunicarse mediante WhatsApp tanto con la escuela como con sus estudiantes, quienes no respetaban el horario habitual de trabajo. 
Revista de la Escuela de Ciencias de la Educación. 2022, Año 18 1(17) 18-31. Enero a junio. Anderete Schwal, M., La pandemia y el año que enseñamos por WhatsApp: el recurso tecnológico más utilizado en las secundarias pobres de Bahía Blanca durante el 2020.

Antes de la pandemia la comunicación se producía dentro de la escuela y en horario escolar, mientras que durante el 2020 los límites temporales del trabajo docente parecieron difuminarse ya que la escuela como espacio físico se convirtió en sus casas.

\section{CONCLUSIÓN}

La desigualdad escolar en las escuelas analizadas se intensificó en tiempos de pandemia, donde la educación a distancia digital fue factible para algunos sectores que tienen los recursos tecnológicos necesarios y se volvió más difícil de lograr para otros que carecen de dichos recursos, profundizando las desigualdades iniciales. Formichella y Krüger (2013) indican que en Argentina las escuelas de gestión privada obtienen mejores resultados que las públicas porque albergan estudiantes con condiciones más favorables para hacer frente a su carrera escolar. Dicha situación que se evidenció en el contexto analizado en el presente trabajo, ya que en las escuelas públicas sus estudiantes no contaron con la cantidad y variedad de recursos tecnológicos que utilizaron en las de gestión privada.

Las estrategias utilizadas por los docentes durante la educación a distancia por pandemia fueron una adaptación a las TIC disponibles, con el objetivo de lograr los mejores resultados posibles. Al analizarlas en forma comparada entre escuelas de diversa pertenencia socio económica, se evidencian las desigualdades educativas. Mientras que en algunas utilizaron plataformas de internet propias donde se conectaban en línea la totalidad de sus estudiantes con sus profesores, en otras los docentes debían dejar fotocopias que las familias retiraban junto con los bolsones de comida.

WhatsApp, además de ser la aplicación para celulares de mensajería instantánea más popular, se convirtió en el recurso tecnológico de comunicación más utilizado en las escuelas estudiadas. Los grupos de WhatsApp se transformaron en aulas virtuales que acercan a los docentes con sus estudiantes, acentuándose su utilización en las escuelas públicas -en especial aquellas de la periferia- y destacándose la predisposición sin límite de horarios del personal docente. No obstante, sus funciones como herramienta educativa para dictar clases no presenciales no pueden compararse con los recursos tecnológicos más avanzados que se utilizan en las escuelas de gestión privada, los cuales permiten un método de educación más detallado y completo mediante plataformas educativas virtuales, donde acceden los alumnos con sus computadoras personales.

\section{REFERENCIAS}

Anderete Schwal, M. (2019). Efectos del sorteo en una escuela secundaria pre universitaria. Revista Espacios en Blanco, 30(1), 159-177. 
Revista de la Escuela de Ciencias de la Educación. 2022, Año 18 1(17) 18-31. Enero a junio. Anderete Schwal, M., La pandemia y el año que enseñamos por WhatsApp: el recurso tecnológico más utilizado en las secundarias pobres de Bahía Blanca durante el 2020.

Anderete Schwal, M. (2020). Las desigualdades educativas durante la pandemia en la educación primaria de Argentina. Revista Andina De Educación, 4(1), 5-10. https://doi.org/10.32719/26312816.2021.4.1.1

Anderete Schwal, M. (2021). Las desigualdades en la educación secundaria argentina durante la pandemia. Márgenes, Revista de Educación de la Universidad de Málaga, 2(2), 42-56

Braslavsky, C. (1985). La discriminación educativa en Argentina. FLACSO.

Cabrera, L.; Pérez, C.N. y Santana, F. (2020). ¿Se Incrementa la Desigualdad de Oportunidades Educativas en la Enseñanza Primaria con El Cierre Escolar por el Coronavirus?, International Journal of Sociology of Education, Special Issue: COVID-19 Crisis and Socioeducative Inequalities and Strategies to Overcome them, 27-52. http://doi.org/10.17583/rise.2020.

Cardini, A.; D'Alessandre, V. y Torre, E. (2020). Educar en tiempos de pandemia. Respuestas provinciales al COVID-19 en Argentina. Cippec.

CEPAL (2020). COVID19 tendrá graves efectos sobre la economía mundial e impactará a los países de América Latina y el Caribe. Comunicado de Prensa. CEPAL. Disponible en: http://bit.ly/2Mf1atj

Chavez, M. (2017). Jóvenes entre el centro y la periferia de la ciudad, del estado y de la academia. CiudaDanías. 1(6), 1-18. Disponible en: http://bit.ly/3byFLWz

Diaz Jatuf, J. (5 y 6 de agosto de 2014). El WhatsApp como herramienta de intervención didáctica para fomentar el aprendizaje cooperativo. $X$ Jornadas de Material Didáctico y Experiencias Innovadoras en Educación Superior, Buenos Aires http://eprints.rclis.org/23597/1/WhatsApp.pdf

(Argentina).

Dussel, I. (2020). La escuela en la pandemia. Reflexiones sobre lo escolar en tiempos dislocados. Práxis Educativa. (15), 1-16. http://doi.org/10.5212/PraxEduc.v.15.16482.090

Dussel, I. y Fuentes Cardona, M. (2021). Los grupos de WhatsApp y la construcción de nuevas ciudadanías en las escuelas. Educação \& Sociedade, (42), 1-18.

Flick, U. (2012). Introducción a la investigación cualitativa. Ediciones Morata.

Formichella, M. y Krüger, N. (2013). El fracaso escolar en el nivel medio argentino: ¿su mayor frecuencia en las escuelas de gestión pública se debe al tipo de gestión? Estudios Económicos Regionales y Sectoriales. 13(3), 127-144

Formichella, M. y Krüger, N. (2019). Condiciones de educabilidad y resultados escolares en barrios vulnerables de la ciudad de Bahía Blanca, Argentina. OBETS, 14(1), 89-118.

Formichella, M. y Krüger, N. (2020). Pandemia y brechas educativas: reflexiones desde la Economía de la Educación. La investigación en ciencias sociales en tiempos de la pandemia por Covid-19. IESS, 168-186. https://ri.conicet.gov.ar/bitstream/handle/11336/109085/CONICET_Digit al_Nro.d76a662b-3c25-4170-aca9-

ba12c4dd7755_A.pdf?sequence=2\&isAllowed=y 
Revista de la Escuela de Ciencias de la Educación. 2022, Año 18 1(17) 18-31. Enero a junio. Anderete Schwal, M., La pandemia y el año que enseñamos por WhatsApp: el recurso tecnológico más utilizado en las secundarias pobres de Bahía Blanca durante el 2020.

Gewerc, A.; Fraga, F. y Rodés, V. (2017). Niños y adolescentes frente a la Competencia Digital. Entre el teléfono móvil, youtubers y videojuegos. Revista Interuniversitaria de Formación del Profesorado, 89(31.2), 171186. https://bit.ly/36aEe5z

Hernández Sampieri, R.; Fernández Collado, C., y Baptista Lucio, P. (2014). Metodología de la investigación. McGraw-Hill.

Kessler, G. (2002). La experiencia escolar fragmentada. Estudiantes y docentes en la escuela media en Buenos Aires. IIPE-UNESCO.

Kessler, G. (2011). Exclusión social y desigualdad inociones útiles para pensar la estructura social argentina? Labvoratorio. Revista de Estudios sobre Cambio Estructural y Desigualdad Social, (24).

Krüger, N. (12-14 de septiembre de 2012). ¿Escuelas pobres para los pobres? Un análisis basado en PISA 2009. Congreso I Jornadas de Jóvenes Investigadores en Educación, FLACSO.

Krüger, N. (2019). La segregación por nivel socioeconómico como dimensión de la exclusión educativa: 15 años de evolución en América Latina. Archivos Analíticos de Políticas Educativas, 27(8), 35-67. https://doi.org/10.14507/epaa.27.3577

Llach, J. (2006). El desafío de la equidad educativa. Granica.

Martínez López, O. (2020). Brecha digital educativa. Cuando el territorio es importante. Sociedad e Infancias, (4), 267-270. https://doi.org/10.5209/soci.69629

Narodowski, M.; Volman, V. y Braga, F. (2020). Dispositivos y medios de comunicación para mantener el vínculo pedagógico en cuarentena. Argentinos por la educación.

Pintrich, P. y Schunck, D. (2006). Motivación en contextos educativos: Teoría, investigaciones y aplicaciones. Pearson Educación.

Pimienta, D. (2007). Brecha digital, brecha social, brecha paradigmática. Funredes.

http://funredes.org/mistica/castellano/ciberoteca/tematica/brecha_paradi gmatica.pdf

Romero, C., Krichesky, G. y Zacarías, N. (2021). "Escuelas WhatsApp y Escuelas Zoom" Desigualdad y segregación educativa durante la pandemia COVID -19 en Argentina. Escuela de Gobierno, Universidad Torcuato di Tella. Documentos de trabajo: https://bit.ly/2REorES

Tiramonti, G. (2004). La trama de la desigualdad educativa. Mutaciones recientes en la escuela media. Manantial.

UNESCO (2020). Consecuencias negativas del cierre de las escuelas. https://es.unesco.org/covid19/educationresponse/consecuencias

UNICEF (2020). La educación frente al Covid-19. Propuestas para impulsar el derecho a la educación durante la emergencia. https://www.unicef.es/educa/biblioteca/la-educacion-frente-al-covid-19

Veleda, C. (2014). Regulación estatal y segregación educativa en la Provincia de Buenos Aires. Cippec. https://core.ac.uk/download/pdf/267852828.pdf 
Revista de la Escuela de Ciencias de la Educación. 2022, Año 18 1(17) 18-31. Enero a junio. Anderete Schwal, M., La pandemia y el año que enseñamos por WhatsApp: el recurso tecnológico más utilizado en las secundarias pobres de Bahía Blanca durante el 2020.

Vivanco, A. (2020). Teleducación en tiempos de COVID-19: brechas de desigualdad. Cienciamérica, http://dx.doi.org/10.33210/ca.v9i2.307

$9(2)$, 166-175. 